\title{
Effect of the new synthetic vitamin E derivative ETS-GS on radiation enterocolitis symptoms in a rat model
}

\author{
SATOSHI SUGITA, MASAFUMI INOMATA, YOHEI KONO, HIDEFUMI SHIROSHITA, \\ TSUYOSHI ETOH, NORIO SHIRAISHI, and SEIGO KITANO
}

Department of Gastroenterological and Pediatric Surgery, Oita University Faculty of Medicine, Yufu, Oita 879-5593, Japan

Received March 16, 2013; Accepted August 28, 2013

DOI: $10.3892 / 01.2013 .1581$

\begin{abstract}
Radiation enterocolitis is a severe adverse event that occurs after radiotherapy for malignant abdominal tumors. In this study, the therapeutic effects of ETS-GS, a novel vitamin E derivative with antioxidative and anti-inflammatory abilities, were examined in a rat model of radiation enterocolitis. The radiation enterocolitis model was created by irradiation of male rats with a single dose of $10 \mathrm{~Gy}$. ETS-GS was administered subcutaneously (10 mg/kg/day) for five consecutive days from two days prior to irradiation. The animals were sacrificed three days after irradiation; following which, ileal tissue samples were analyzed for macroscopic and histological findings, presence of apoptosis, degree of oxidative stress and inflammation. In the irradiated group, severe erosion was observed in the small intestine in addition to necrosis of the mucosal layer, swelling and invasion of inflammatory cells of the submucosal layer, and shortening of the crypts. In irradiated rats that received ETS-GS, mucosal injury in the small intestine was milder compared with that of irradiated rats that received no ETS-GS. In addition, ETS-GS decreased apoptosis in the small intestine and reduced the activity of myeloperoxidase and malondialdehyde, which are markers for inflammation and oxidative stress. ETS-GS with antioxidant activity has a therapeutic effect on the symptoms of radiation enterocolitis in a rat model.
\end{abstract}

\section{Introduction}

Radiation enterocolitis is a severe adverse event resulting from radiation therapy for abdominal and pelvic malignancies $(1,2)$. As administration of radiation therapy has increased in recent years, the incidence of radiation enterocolitis has also increased (3). Drug therapies for the treatment of

Correspondence to: Dr Satoshi Sugita, Department of Gastroenterological and Pediatric Surgery, Oita University Faculty of Medicine, 1-1 Idaigaoka, Hasama-machi, Yufu, Oita 879-5593, Japan

E-mail: s-sugita@oita-u.ac.jp

Key words: radiation enterocolitis, vitamin E derivative, apoptosis radiation enterocolitis include antispasmodic, antidiarrheal, steroidal $(4,5)$ and nonsteroidal anti-inflammatory agents, such as sulfasalazine (4) and aspirin (6,7). In cases refractory to treatment using these drugs, surgical treatment is often considered. Therefore, the development of new and more effective drugs for the treatment of radiation enterocolitis is desirable.

Certain studies have suggested that radiation enterocolitis is caused by oxidative stress, as antioxidants including aminoguanidine, octreotide and glutamine have been shown to improve radiation enterocolitis in animal models (8-10). In this study, a new antioxidant agent, ETS-GS $(\gamma$-L-glutamyl-S-[2[[[3,4-dihydro-2,5,7,8-tetramethyl-2-(4,8,12-trimethyltri-decyl)2H-1-benzopyran-6-yl]oxy] carbonyl]-3-oxo-3-[(2-sulfoethyl) amino]propyl]-L-cysteinylglycine sodium salt), was developed for the treatment of radiation enterocolitis. This agent is soluble and stable in water, and has high antioxidative and anti-inflammatory activity. Several studies have validated the antioxidative and anti-inflammatory effects of this agent (11-15).

In the present study, the therapeutic effects of this new vitamin E derivative, ETS-GS, were examined in a rat model of radiation-induced enterocolitis.

\section{Materials and methods}

Chemicals. The vitamin E derivative ETS-GS consists of chemically linked vitamin E, glutathione, taurine and malefic acid. The sterile, aqueous solution of ETS-GS used in this experiment was provided by Oga Research Inc. (Osaka, Japan; Fig. 1).

Animals. Six-week-old male Sprague-Dawley rats (Kyudo, Saga, Japan) weighing 150-250 g $(n=27)$ were used in all experiments. The rats were housed in cages controlled for temperature $\left(22 \pm 2^{\circ} \mathrm{C}\right)$, humidity and lighting (12-h light/dark cycle), with free access to water and food. The study protocol was approved by the Animal Ethics Committee of Oita University Faculty of Medicine (Yufu, Japan).

Experimental protocol. Rats were randomly divided into three groups ( $\mathrm{n}=9$ per group) according to treatment. The untreated group received no radiation and no treatment, the irradiated (IR) group received abdominal radiation, and the IR + ETS-GS group received abdominal radiation and ETS-GS treatment. ETS-GS $(10 \mathrm{mg} / \mathrm{kg}$ dissolved in $0.9 \% \mathrm{NaCl})$ was administered 


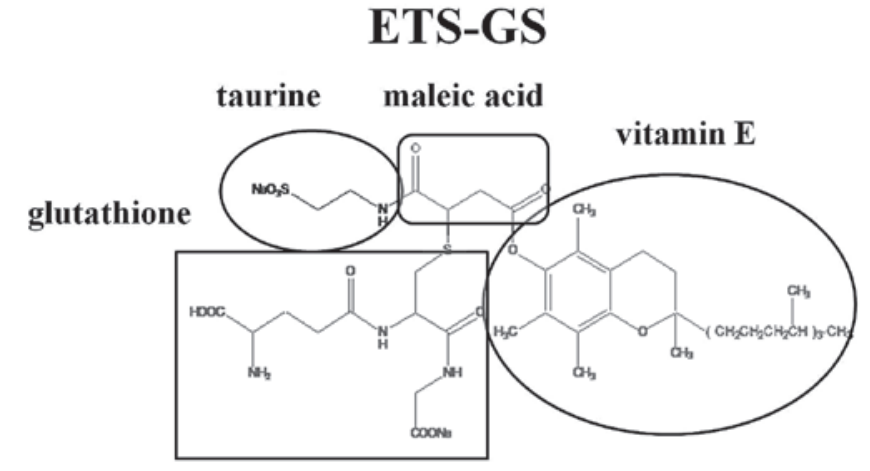

Figure 1. Structure of ETS-GS ( $\gamma$-L-Glutamyl-S-[2-[[[3,4-dihydro-2,5,7,8-tetramethyl-2-(4,8,12-trimethyl-tri-decyl)-2H-1-benzopyran-6-yl]oxy] carbonyl]-3-oxo-3-[(2-sulfoethyl)amino]propyl]-L-cysteinylglycine sodium salt).

subcutaneously for five consecutive days commencing two days prior to radiation exposure. The dose of ETS-GS was determined according to the protocol in a previous study using this rat model (12-14).

Prior to irradiation, rats were anesthetized by intraperitoneal injection of sodium pentobarbital (Nembutal; Dainippon Sumitomo Parma Co., Ltd., Japan) at a dose of $50 \mathrm{mg} / \mathrm{kg}$ body weight, and placed in the supine position. They were then irradiated with a single dose of 10 Gy to the whole abdomen using a Gammacell 40 Extractor (Atomic Energy of Canada Ltd., Chalk River, ON, Canada). Modified lead plates were used to shield the head and chest of the rats during irradiation. Three days after irradiation, rats were sacrificed humanely according to the Institutional Animal Care and Use Committee guidelines of Oita University. The terminal ilea were harvested for analysis, and a portion was fixed in buffered formalin for histopathological examination. The remainder tissue was stored at $-80^{\circ} \mathrm{C}$ for biochemical analysis.

Histopathological analysis. After washing with saline solution, ileal tissue specimens were fixed in $10 \%$ buffered formaldehyde and embedded in paraffin, and 5- $\mu \mathrm{m}$ sections were prepared using a microtome. Sections were stained with hematoxylin and eosin and analyzed for morphological changes.

Detection of DNA fragmentation. Using the terminal deoxynucleotidyl transferase (dUTP) nick-end labeling (TUNEL) method, DNA fragmentation was detected under a fluorescent microscope, and flow cytometry was performed promptly. Highly sensitive and specifically labeled fluorescein dUTP is labeled on the free 3'-OH end of fragmented DNA of the cell, after which terminal transferase causes apoptosis. Staining was performed using an MK500 In situ Apoptosis Detection kit (Takara Bio Inc., Otsu, Japan) according to the manufacturer's instructions. In total, 1000 cells, including cells positive for TUNEL, were counted in 10 random high-power fields (x400 magnification), and the percentage of TUNEL-positive cells was used to calculate the apoptotic index $(16,17)$.

Caspase-3/7 activity. Caspases are a group of cysteine proteases that constitute a signaling pathway which causes cells to undergo apoptosis. Caspase-3/7, also known as effector

A

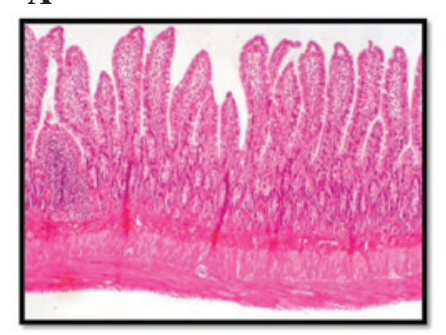

B

C

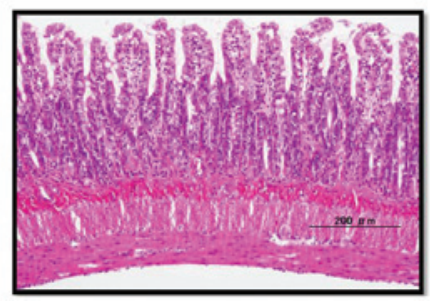

Figure 2. Histopathological changes in ileal tissue after irradiation. Ileal tissues were fixed, embedded, sectioned and stained with hematoxylin and eosin. No histological alternations were observed in (A) the untreated group, whereas severe erosion, necrosis of the mucosal layer, swelling and invasion of inflammatory cells of the submucosal layer, and shortening of the crypts were observed in (B) the irradiated (IR) group. Attenuated irradiated changes were observed in (C) the IR + ETS-GS group. Magnification, x200.

A
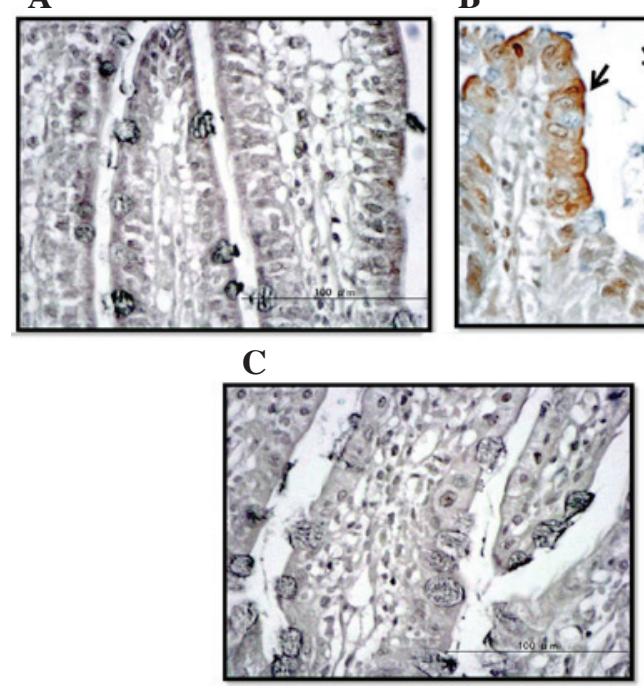

Figure 3. Terminal deoxynucleotidyl transferase nick-end labeling staining of an ileal section. (B) Representative histological section from the irradiated (IR) group, in which numerous dyed apoptotic cells are visible (arrows). By contrast, in (A) the untreated group and (C) the IR + ETS-GS group, almost no apoptotic cells were present. Magnification, $\mathrm{x} 400$.

caspases, are activated by initiator caspases. They work to disassemble other intracellular proteins and increase apoptosis; in cells undergoing apoptosis, caspase-3/7 activity is enhanced $(18,19)$. Caspase- $3 / 7$ activity was measured in this study using a Caspase-Glo ${ }^{\circledR}$ 3/7 assay kit (Promega Co., Madison, WI, USA) according to the manufacturer's instructions.

Biomarker assays. Intestinal myeloperoxidase (MPO) activity was assayed using a rat MPO enzyme-linked immunosorbent 


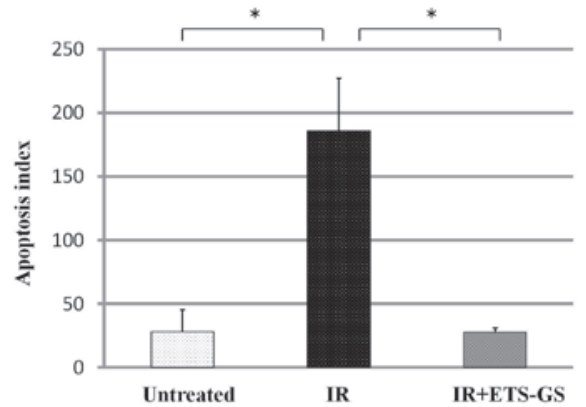

Figure 4. Number of apoptotic cells. Terminal deoxynucleotidyl transferase nick-end labeling (TUNEL)-positive cells were counted over 10 fields selected randomly in the field of view, which included 1000 cells, using an optical microscope (magnification, $\mathrm{x} 400$ ). Results are expressed as the mean \pm standard deviation $\left(\mathrm{n}=9\right.$ per group, $\left.{ }^{*} \mathrm{P}<0.05\right)$

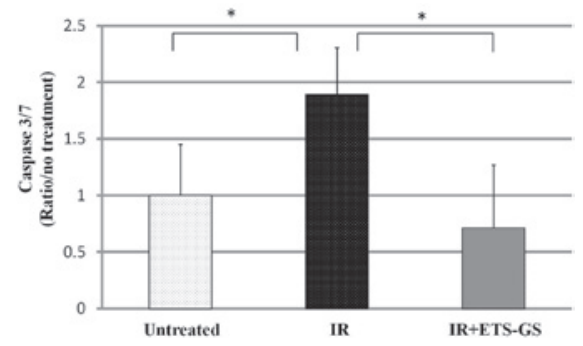

Figure 5. Effect of ETS-GS on caspase-3/7 levels in the ileal tissue of rats following irradiation. Caspase-3/7 levels in the ileal tissues were measured three days after irradiation. Results are expressed as the mean \pm standard deviation $\left(\mathrm{n}=9\right.$ per group, $\left.{ }^{*} \mathrm{P}<0.05\right)$.

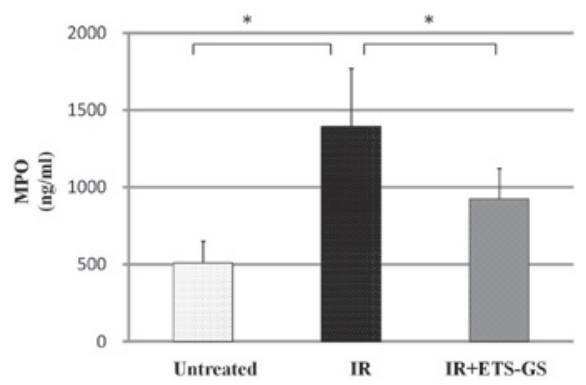

Figure 6. Effect of ETS-GS on myeloperoxidase (MPO) levels in the ileal tissue of rats after irradiation. The activity of MPO in the ileal tissues was examined three days after irradiation. Results are expressed as the mean \pm standard deviation ( $\mathrm{n}=9$ per group, ${ }^{*} \mathrm{P}<0.05$ ).

assay (ELISA) kit (Hycult ${ }^{\circledR}$ Biotech, Uden, The Netherlands) according to the manufacturer's instructions. MPO is a peroxidase enzyme most abundant in neutrophil granulocytes (20). Malondialdehyde (MDA) in the ileal tissue was assayed to assess the degree of oxidative stress using a commercial kit for thiobarbituric acid reactive substances (Northwest Life Science Specialties LLC, Vancouver, WA, USA). Absorbance at $532 \mathrm{~nm}$ was determined using an ELISA plate reader (Bio-Rad Laboratories, Hercules, CA, USA).

Statistical analysis. All data are expressed as the mean \pm standard deviation. One-way analysis of variance was used for statistical analysis, and $\mathrm{P}<0.05$ was considered to indicate a statistically significant difference. The Dr. SPSS II statistical

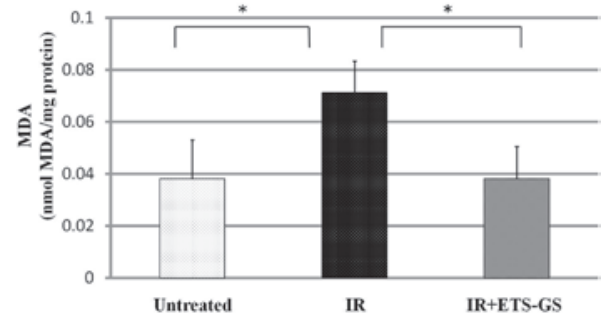

Figure 7. Effect of ETS-GS on malondialdehyde (MDA) levels in the ileal tissue of rats after irradiation. MDA levels in the ileal tissues were measured three days after irradiation. Results are expressed as the mean \pm standard deviation $\left(\mathrm{n}=9\right.$ per group, $\left.{ }^{*} \mathrm{P}<0.05\right)$

software (SPSS, Inc., Chicago, IL, USA) was used to evaluate the data.

\section{Results}

Macroscopic and histopathological findings. In this study, no treatment-related mortality occurred in any rats. In the IR group, histological examination showed severe erosion, necrosis of the mucosal layer, swelling and invasion of inflammatory cells of the submucosal layer, and shortening of the crypts in the ileum (Fig. 2). In the IR + ETS-GS group, ileal injury was improved compared with that of the IR group. In addition, rats administered only ETS-GS were histologically similar to the untreated group in the preliminary experiment (data not shown).

Assessment of apoptosis using the TUNEL method and caspase-3/7 activity. TUNEL staining and caspase-3/7 assay were used to evaluate apoptosis in ileal tissue specimens. In the IR group, numerous dyed apoptotic cells were confirmed in the mucosal layer of the intestine. By contrast, very few dyed apoptotic cells were observed in the specimens obtained from the untreated and IR + ETS-GS groups (Fig. 3). The apoptotic index of the IR group was significantly higher than that of the untreated group $(186 \pm 41.1$ vs. $28.3 \pm 17.6, \mathrm{P}<0.05)$ (Fig. 4). In the IR + ETS-GS group, the apoptotic index was lower than that in the IR group $(27.7 \pm 3.5$ vs. $186 \pm 41.1, \mathrm{P}<0.05)$.

In the IR group, intestinal caspase-3/7 activity was found to be significantly increased compared with that in the untreated group ( $1.89 \pm 0.42$ vs. $1.00 \pm 0.45, \mathrm{P}<0.05)$; however, ETS-GS significantly decreased this activity $(0.71 \pm 0.56)$ (Fig. 5).

MPO activity. The activity of intestinal MPO was measured to assess the degree of inflammation. Intestinal MPO activity in the IR group was significantly higher than that in the untreated group (1394.0 \pm 375.6 vs. $510.3 \pm 141.8 \mathrm{ng} / \mathrm{ml}, \mathrm{P}<0.05)$. In the IR + ETS-GS group, MPO activity was significantly decreased compared with that in the IR group $(925.0 \pm 196.7$ vs. $1394.0 \pm 375.6 \mathrm{ng} / \mathrm{ml}, \mathrm{P}<0.05$ ) (Fig. 6).

MDA assay. The activity of intestinal MDA was measured to assess the degree of oxidative stress. MDA is formed by oxidation of lipids and is an indicator of oxidative stress (21). In the IR group, the intestinal MDA level was found to be significantly increased compared with that in the untreated group ( $0.07 \pm 0.01$ vs. $0.04 \pm 0.02, \mathrm{P}<0.05)$. Treatment with ETS-GS 
significantly decreased the MDA level compared with that of the IR group $(0.04 \pm 0.01$ vs. $0.07 \pm 0.01, \mathrm{P}<0.05)$ (Fig. 7).

\section{Discussion}

To the best of our knowledge, this is the first study demonstrating the effect of the new vitamin E derivative ETS-GS on radiation enterocolitis in rats. Histopathological analysis showed that administration of ETS-GS decreased the degree of mucosal erosion and necrosis, swelling and invasion of inflammatory cells of the submucosal layer, and shortening of the crypts caused by acute radiation enterocolitis. This biological activity was associated with caspase-3/7 levels, apoptotic index, and MPO and MDA levels in ileal tissue specimens of rats treated by administration of ETS-GS.

Several animal models of radiation enterocolitis have been developed. Mylonas et al prepared a model by abdominal irradiation of a single dose of $11 \mathrm{~Gy}$ in rats (22), while Wang et al created a model by irradiating once daily $4.2 \mathrm{~Gy}$ for 16 days (23). In this study, the radiation enterocolitis model was created by irradiation of a single dose of $10 \mathrm{~Gy}$ to male rats. This method was successful in inducing radiation enterocolitis. Examinations were performed with future clinical application in mind. Huang et al administered aminoguanidine prior to irradiation when examining the effects of this agent on small bowel damage in rats, to demonstrate its prophylactic effect (8). By contrast, Emami et al administered aminoguanidine after irradiation, with the intention of showing its therapeutic effect (24). In the present study, the drug was administered prior to and after irradiation, consecutively for five days. As a result, the onset of radiation enterocolitis was significantly suppressed. In addition, a similar effect was observed after administration of ETS-GS (10 mg/kg), with no significant toxicity, as observed in previous studies (12-14).

Abdominal irradiation causes inflammation of the intestinal tract with submucosal edema, hyperemia and infiltration of the lamina propria due to the activation of inflammatory cells (24). Radiation energy absorbed by the body is supplied to biopolymers, such as nucleic acids and proteins, as well as to in vivo water molecules. These molecules are then ionized or energized, thereby inducing radiation damage $(25,26)$. Radiation injury can be caused by direct or indirect action (27). Direct action causes damage directly to the biopolymers, and indirect action causes ionization and dissociation of water molecules. With indirect action, free radicals and reactive oxygen species are formed through secondary chain reactions, inducing apoptosis and tissue damage. Furthermore, Hepgül et al and Mollà et al reported the involvement of oxidative stress in radiation-induced enterocolitis $(28,29)$. MPO exists in abundance in neutrophils and is used as a marker for the detection of neutrophil accumulation within inflamed tissue (20). MDA is formed by oxidization of lipids and is an indicator of oxidative stress (21). Caspase-3/7 is activated by initiator caspases, such as caspase-8/9; these caspases break down proteins within cells and trigger apoptosis, which is why they are used as markers of apoptosis $(18,19)$. In this experiment, dysfunction of the ileal mucous membrane as a result of radiation exposure was inhibited through ETS-GS administration, and ileal MPO, MDA and caspase-3/7 protein activity was significantly decreased. Based on these findings, we suggest that oxidative stress, which occurs due to radiation enterocolitis, is impeded by administration of ETS-GS, and tissue damage is prevented through inhibition of apoptosis.

ETS-GS is highly soluble in water and has both antioxidant and anti-inflammatory actions, including suppression of MDA, interleukin-6 and tumor necrosis factor- $\alpha(13,14)$. As ETS-GS dissolves easily in water, administration not only by subcutaneous injection but also by intravenous injection and drip infusion may be possible. Intravenous injection of ETS-GS has been shown to improve lipopolysaccharide-induced acute lung and liver injury and renal ischemia reperfusion in rat models, with no reports of apparent side-effects $(13,14)$. Numerous reported therapeutic agents for the treatment of radiation enteritis must be administered orally (30-32). ETS-GS has the advantage that it may be administered intravenously to patients with radiation-induced enterocolitis for whom oral administration is not possible.

The present study is an animal experiment using rat models; however, the results for clinical application were encouraging. For clinical purposes, the mechanism of the agent must be clarified in more detail, and the presence or absence of side-effects must be confirmed. Radiation is utilized in the treatment of malignant tumors; the effects of ETS-GS administration on these tumors and its interaction with radiation therapy also require clarification. A previous study demonstrated the antiproliferative effects of a new $\alpha$-lipoic acid derivative, DHL-HisZnNa, with strong antioxidant action similar to that of ETS-GS, in HT29 human colon cancer cells in vitro (33). Similar effects are predicted for ETS-GS, and future studies must verify this hypothesis.

In conclusion, this study demonstrated that a new synthetic vitamin E derivative, ETS-GS, has therapeutic effects against the intestinal damage caused by abdominal irradiation, through reduction of the inflammatory response, apoptosis and oxidative stress.

\section{Acknowledgements}

The authors would like to thank Mr. Kazumi Ogata (Oga Research, Inc., Osaka, Japan) for donating ETS-GS; and Dr Masatsugu Moriyama, Dr Yoko Komori, Ms. Yuiko Aso, Ms. Seiko I, Ms. Mayumi Takeda, Ms. Hiroko Taguchi, Mr. Hiroaki Kawazato and Ms. Akio Yasuda for their helpful advice on hematoxylin and eosin staining, thoughtful comments and technical assistance. This study was supported in part by Grants-in-Aid for Scientific Research (C) from the Japan Society for the Promotion of Science (nos. 23591967 and 24791386).

\section{References}

1. Kan S, Chun M, Jin YM, et al: A rat model for radiation-induced proctitis. J Korean Med Sci 15: 682-689, 2000.

2. Yeoh E and Horowitz M: Radiation enteritis. Br J Hosp Med 39: 498-504, 1988

3. Ooi BS, Tjandra JJ and Green MD: Morbidities of adjuvant chemotherapy and radiotherapy for resectable rectal cancer: an overview. Dis Colon Rectum 42: 403-418, 1999.

4. Kochhar R, Patel F, Dhar A, et al: Radiation-induced proctosigmoiditis. Prospective, randomized, double-blind controlled trial of oral sulfasalazine plus rectal steroids versus rectal sucralfate. Dig Dis Sci 36: 103-107, 1991. 
5. Denton AS, Andreyev HJ, Forbes A and Maher EJ: Systematic review for non-surgical interventions for the management of late radiation proctitis. Br J Cancer 87: 134-143, 2002.

6. Mennie AT, Dalley VM, Dinneen LC and Collier HO: Treatment of radiation-induced gastrointestinal distress with acetylsalicylate. Lancet 2: 942-943, 1975.

7. Stryker JA, Demers LM and Mortel R: Prophylactic ibuprofen administration during pelvic irradiation. Int J Radiat Oncol Biol Phys 5: 2049-2052, 1979.

8. Huang EY, Wang FS, Lin IH and Yang KD: Aminoguanidine alleviates radiation-induced small-bowel damage through its antioxidant effect. Int J Radiat Oncol Biol Phys 74: 237-244, 2009.

9. Abbasoğlu SD, Erbil Y, Eren T, Giriş M, et al: The effect of heme oxygenase-1 induction by octreotide on radiation enteritis. Peptides 27: 1570-1576, 2006.

10. Giriş M,Erbil Y,Oztezcan S, et al: The effect of heme oxygenase-1 induction by glutamine on radiation-induced intestinal damage: the effect of heme oxygenase-1 on radiation enteritis. Am J Surg 191: 503-509, 2006.

11. Kono Y, Inomata M, Hagiwara S, et al: A newly synthetic vitamin E derivative, E-Ant-S-GS, attenuates lung injury caused by cecal ligation and puncture-induced sepsis in rats. Surgery 151: 420-426, 2012.

12. Koga H, Hagiwara S, Inomata M, et al: Vitamin E derivative ETS-GS reduces liver ischemia-reperfusion injury in rats. J Surg Res 175: 118-122, 2012

13. Hagiwara S, Koga H, Iwasaka H, et al: ETS-GS, a new anti-oxidative drug, protects against lipopolysaccharide-induced acute lung and liver injury. J Surg Res 171: 734-741, 2011.

14. Hagiwara S, Koga H, Iwasaka H, et al: ETS-GS, a new antioxidant, ameliorates renal ischemia-reperfusion injury in a rodent model. J Surg Res 171: 226-233, 2011.

15. Hiratsuka T, Inomata M, Hagiwara S, et al: Bolus injection of newly synthesized vitamin E derivative ETS-GS for the treatment of acute severe ulcerative colitis in a mouse model. Int J Colorectal Dis 28: 305-311, 2013.

16. Kikuchi A and Nishikawa T: Apoptotic and proliferating cells in cutaneous lymphoproliferative diseases. Arch Dermatol 133: 829-833, 1997.

17. El-Domyati M, Abo-Elenin M, El-Din WH, et al: Expression of apoptosis regulatory markers in the skin of advanced hepatitis-C virus liver patients. Indian J Dermatol 57: 187-193, 2012.

18. Kothakota S, Azuma T, Reinhard C, et al: Caspase-3-generated fragment of gelsolin: effector of morphological change in apoptosis. Science 278: 294-298, 1997.

19. Salvesen GS and Riedl SJ: Caspase mechanisms. Adv Exp Med Biol 615: 13-23, 2008
20. Liu SH, Ma K, Xu XR and Xu B: A single dose of carbon monoxide intraperitoneal administration protects rat intestine from injury induced by lipopolysaccharide. Cell Stress Chaperones 15: 717-727, 2010.

21. Nielsen F, Mikkelsen BB, Nielsen JB, et al: Plasma malondialdehyde as biomarker for oxidative stress: reference interval and effects of life-style factors. Clin Chem 43: 1209-1214, 1997.

22. Mylonas PG, Matsouka PT, Papandoniou EV, et al: Growth hormone and insulin-like grouth factor I protect intestinal cells from radiation induced apoptosis. Mol Cell Endocrinol 160: $115-122,2000$

23. Wang J, Zheng H, Sung CC, et al: The synthetic somatostatin analogue, octreotide, ameliorates acute and delayed intestinal radiation injury. It J Radiat Oncol Biol Phys 45: 1289-1296, 1999.

24. Emami B, Lyman J, Brown A, et al: Tolerance of normal tissue to therapeutic irradiation. Int J Radiat Oncol Biol Phys 21: 109-122, 1991.

25. Nair CK, Parida DK and Nomura T: Radioprotectors in radiotherapy. J Radiat Res 42: 21-37, 2001.

26. Kilciksiz S, Demirel C, Erdal N, et al: The effect of $\mathrm{N}$-acetylcysteine on biomarkers for radiation-induced oxidative damage in a rat model. Acta Med Okayama 62: 403-409, 2008.

27. Perez CA. The Discipline of Radiation Oncology. In: Principles and Practice of Radiation Oncology. Perez CA, Brady LW, Halperin EC, Schmidt-Ullrich R (eds). 4th edition. Lippincott Williams \& Wilkins, Philadelphia, pp9-10, 2003.

28. Hepgül G, Tanrikulu S, Unalp HR, et al: Preventive effect of pentoxifylline on acute radiation damage via antioxidant and anti-inflammatory pathways. Dig Dis Sci 55: 617-625, 2010.

29. Mollà M, Gironella M, Salas A, et al: Protective effect of superoxide dismutase in radiation-induced intestinal inflammation. Int J Radiat Oncol Biol Phys 61: 1159-1166, 2005.

30. Membrive Conejo I, Reig Castillejo A, Rodríguez de Dios N, et al: Prevention of acute radiation enteritis: efficacy and tolerance of glutamine. Clin Transl Oncol 13: 760-763, 2011.

31. Takeda T, Kamiura S and Kimura T: Effectiveness of the herbal medicine daikenchuto for radiation-induced enteritis. J Altern Complement Med 14: 753-755, 2008

32. Zimmerer T, Böcker U, Wenz F and Singer MV: Medical prevention and treatment of acute and chronic radiation induced enteritis - is there any proven therapy? a short review. Z Gastroenterol 46: 441-448, 2008.

33. Kono Y, Inomata M, Hagiwara S, et al: Antiproliferative effects of a new $\alpha$-lipoic acid derivative, DHL-HisZnNa, in HT29 human colon cancer cells in vitro. Expert Opin Ther Targets 16 (Suppl 1): S103-S109, 2012. 